
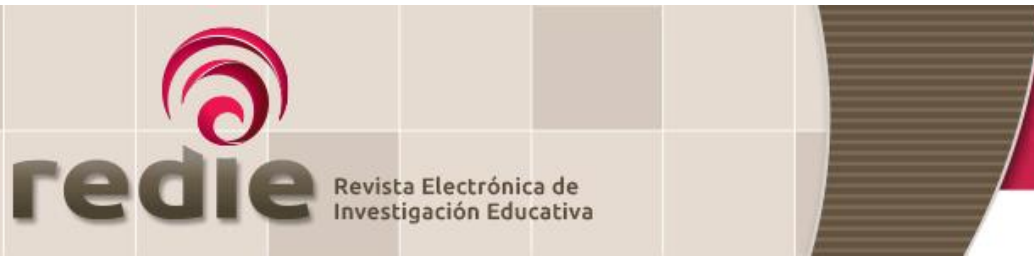

\title{
Estudio de correlación entre la simulación telemática y las destrezas lingüísticas en inglés
}

\section{A Correlation Study between Telematic Simulation and English Language Skills}

María Laura Angelini (1) marialaura.angelini@ucv.es

Amparo García-Carbonell (2) agarciac@upvnet.upv.es

Nieves Martínez-Alzamora (2) nalzamor@eio.upv.es

(1) Universidad Católica de Valencia "San Vicente Mártir"

(2) Universitat Politècnica de València

(Recibido: 26 de febrero de 2015; Aceptado para su publicación: 8 de septiembre de 2015)

Cómo citar: Angelini, M. L., García-Carbonell, A. y Martínez-Alzamora, N. (2017). Estudio de correlación entre la simulación telemática y las destrezas lingüísticas en inglés. Revista Electrónica de Investigación Educativa, 19(1), 141-156. Recuperado de https://doi.org/10.24320/redie.2017.19.1.1100

\section{Resumen}

El artículo trabajo presenta un estudio cuantitativo discreto mediante un estudio de correlación entre la simulación telemática y el progreso en destrezas lingüísticas en inglés. Persigue dos objetivos: comprobar si la simulación telemática constituye una herramienta eficaz en la adquisición de la lengua inglesa y contribuir a la investigación que parte desde la práctica en el aula de lenguas para fines específicos. El estudio se realizó con una población de 50 estudiantes de la carrera de Ingeniería en Telecomunicación de la Universitat Politècnica de València. Se han estimado los coeficientes de correlación entre las variables utilidad, nivel pre-tratamiento, progreso en destrezas, factores que influyen en el progreso, contribución de las partes de la simulación telemática y progreso en capacidades y actitud, y se han determinado los intervalos de confianza al 95\% por el método bootstrap. Los resultados obtenidos confirman que existe una fuerte correlación entre la simulación telemática y el progreso en destrezas lingüísticas como también entre la simulación telemática y el progreso en habilidades transversales o profesionales.

Palabras clave: Simulación y juego, aprendizaje a través de la experiencia, simulación telemática, metodologías activas, aprendizaje de lenguas.

\section{Abstract}

This paper offers a discrete quantitative study by means of a correlation study between telematic simulation and progress in English language skills. This pursues a double aim: to test whether telematic simulation is an efficient tool in English language acquisition, and contribute to research based on language classroom practice for specific purposes. The study was carried out 
with an experimental population of 50 students of the telecommunications engineering major in the Universitat Politècnica de Valencia. The study estimated the correlation coefficients between the variables usefulness, pre-treatment level, skill progress, factors that affect progress, the contribution of the parts of telematic simulation and the progress in ability and attitude. Confidence levels of $95 \%$ were determined with the bootstrap method. The results obtained confirm a strong correlation between telematic simulation and progress in language skills, and between telematic simulation and progress in transversal or professional skills.

Keywords: Simulation and gaming; experiential learning; telematic simulation; active learning, language learning

\section{Introducción}

El manejo de lenguas se presenta como un requisito indispensable del mundo laboral actual. Desde el Espacio Europeo de Educación Superior (EEES) se implementa una política lingüística global que conlleva una transformación en el paradigma educativo de lenguas para fines específicos con el objetivo de garantizar un tratamiento efectivo de la enseñanza y aprendizaje de lenguas. La Organización para la Cooperación Económica y el Desarrollo (OECD) hace hincapié en la necesidad de profesionales competentes en formación, habilidades, valores y un gran dominio de lenguas. Por lo que las universidades se convierten en la plataforma para alcanzar no sólo un nivel de suficiencia lingüística, sino que las competencias transversales formen parte del proceso de enseñanza-aprendizaje.

La población universitaria que participó en esta investigación cursa estudios de ingeniería, para los que la organización ABET (Accreditation Board for Engineering and Technology), responsable de la acreditación de calidad de programas de ingeniería y ciencia aplicada, identifica seis destrezas profesionales relacionadas con el proceso y el conocimiento. Las competencias de comunicación, trabajo en equipo, ética profesional, conocimientos específicos dentro de un contexto global y social, un aprendizaje continuo y el conocimiento de asuntos de interés actual son necesarios para satisfacer al sector productivo (Shuman, Besterfield-Sacre y McCourty, 2005). El perfil profesional al que aspiran los futuros egresados de la población universitaria que participó en este estudio coincide con este planteamiento y justifica la implementación de la metodología de la simulación y juego y la simulación telemática para la enseñanza-aprendizaje de la lengua inglesa.

Si bien la metodología de la simulación y juego está en crecimiento en España, especialistas con una larga trayectoria en simulación y juego a nivel internacional (Bredemeier y Greenblat, 1981; Crookall y Arai, 1995; Cherryholmes, 1966; De Caluwé, Hofstede y Peters, 2008; Duke, 1974; Goodman, 1984, 1995; Gredler, 1992; Greenblat, 1975; Greenblat y Duke, 1975, 1981; Lederman, 1983, 1992; Lederman y Stewart, 1991; Shubik, 1994; Taylor y Walford, 1978 y Wolfe y Crookall, 1998; entre otros), coinciden en la eficacia de esta metodología en la enseñanza universitaria. La simulación y juego plantea un escenario de actuación con temas específicos de alguna especialidad académica o de interés global. El alumnado, dividido en equipos de trabajo, lleva a cabo el estudio de ese escenario, adopta y negocia posturas, toma decisiones, resuelve la situación presentada y reflexiona sobre la experiencia. La metodología de la simulación y juego, por lo tanto, a través de escenarios de actuación, propicia la adquisición de conocimientos especializados, entre ellos los lingüísticos, al tiempo que permite interactuar, comunicar, liderar, negociar o trabajar en equipo, entre otros, en contextos reales (Vernon, 2006; Blum y Scherer, 2007; Crookall, 2010; García-Carbonell y Watts, 2012). De esta manera, se logra integrar la

\footnotetext{
1 ABET es una organización no gubernamental reconocida, sin ánimo de lucro, dedicada a la acreditación de programas de educación universitaria o terciaria en Ciencias Aplicadas, Ciencias de la Computación, Ingeniería y Tecnología. La acreditación ABET certifica que el plan de estudios forma a sus titulados en las competencias requeridas por la profesión de acuerdo a ciertos estándares de calidad.
} 
materia especializada y el desarrollo de las competencias profesionales (Duke y Geurts, 2004; Kriz y Hense, 2006; Druckman y Mason; 2008; Klabbers, 2009; Hofstede, Caluwé y Peters, 2010; Angelini, García-Carbonell y Watts, 2014).

Por otro lado, el modelo de aprendizaje circular promovido por Kolb (1984) y Kolb y Kolb (2005a, 2005b, 2009), responde al modelo y principios de la simulación y juego (García-Carbonell, Watts y Andreu-Andrés, 2012). Ambos modelos subrayan la importancia de un aprendizaje basado en la experiencia. Se reflexiona y conceptualiza sobre una experiencia concreta vivida y el conocimiento se vuelve a aplicar a nuevas situaciones. En este caso, la simulación telemática ICONS $^{2}$ (International Communication and Negotiation Simulation) ofrece un aprendizaje experiencial y desarrolla la competencia comunicativa a la vez que las competencias transversales.

La efectividad de la simulación telemática en la enseñanza-aprendizaje de lenguas queda recogida en diferentes estudios que corroboran los beneficios de este modelo pedagógico en el aprendizaje del inglés, de sus estructuras gramaticales y sus expresiones lingüísticas dentro del contexto de una simulación: Angelini (2012), Angelini y García-Carbonell (2014), Asal y Blake (2006), Crookall y Oxford (1990); Crookall y Arai (1995), DeGarmo (2006), Ekker (2000, 2004), Ekker y Eidsmo (2006), Ekker y Sutherland (2005, 2009), García-Carbonell (1998), García-Carbonell y Watts (2012), García-Carbonell, Andreu-Andrés y Watts (2014), García-Carbonell, Watts y Andreu-Andrés (2012), Garrido-Iñigo (2012), Halleck y Coll-García (2011), Lay y Smarick (2006), Rising (1999, 2009), Watts, García-Carbonell y Rising (2011) y Wedig (2010), entre otros.

Crookall y Oxford (1990a), García-Carbonell (1998), García-Carbonell y Watts (2012), MacDonald, García-Carbonell y Carot $(2013)$, Rising $(1999,2009)$ y Watts et al. (2011) coinciden en que al utilizar simulaciones se estimula la necesidad de interacción debido a su carácter comunicativo; se potencia el trabajo colaborativo para resolver las situaciones que se plantean y se respetan las necesidades lingüísticas de cada sujeto a fin de que los participantes hagan uso de una amplia gama de registros lingüísticos, vocabulario y expresiones propias del inglés dentro de un entorno específico.

La presente investigación, por tanto, trata de alcanzar un doble objetivo: Primero, comprobar la hipótesis que se plantea: la simulación telemática es efectiva para el aprendizaje del inglés. Рara ello, se lleva a cabo un análisis del progreso y satisfacción con la simulación telemática posttratamiento mediante el estudio de las respuestas a 46 ítems que conforman un cuestionario tipo Likert. Se estudia la relación existente entre el nivel previo del alumnado, aquello en lo que ha progresado, los factores que considera han funcionado mejor, el modelo de aprendizaje que ha funcionado mejor, las capacidades adquiridas y su cambio de actitud; el segundo objetivo es contribuir a la investigación que nace desde el aula y que, según Morales Vallejo (2010, 2012), trata de acercar la investigación y la práctica profesional docente. En definitiva, perseguir un paradigma de enseñanza-aprendizaje de lenguas activo en ingeniería, construido alrededor de la simulación y juego y la integración horizontal y vertical de las competencias específicas y transversales.

\footnotetext{
${ }^{2}$ El Proyecto Icons crea simulaciones a gran escala con escenarios que describen situaciones reales. Entre sus objetivos se encuentra que los participantes interactúen entre sí de forma cooperativa y colaborativa, que alcancen un conocimiento mayor de las situaciones propuestas a través de la experiencia y refuercen sus habilidades para tomar decisiones, negociar y desarrollar el pensamiento crítico, entre otras.
} 


\section{Metodología}

Este trabajo forma parte de un estudio más amplio que analiza la mejora en las destrezas de producción en lengua inglesa mediante la utilización de la simulación y juego como metodología de enseñanza y aprendizaje. En este caso, se presentan los resultados de un análisis cuantitativo discreto intragrupal de las variables que conforman el cuestionario. En el estudio de campo interviene una población experimental de 50 sujetos que pertenecen a la Escuela Técnica Superior de Ingenieros de Telecomunicación de la Universitat Politècnica de València (España). Son estudiantes de lengua inglesa y han participado en una simulación telemática a través del proyecto ICONS, durante los cursos académicos 2011-12 y 2012-13. La investigación presenta un diseño cuasi-experimental donde la población presenta perfiles similares en formación académica y en conocimientos de inglés. Todos están matriculados en la asignatura cuatrimestral Inglés Avanzado y acreditan conocimientos de inglés nivel B1.

El análisis del progreso post-tratamiento en lengua inglesa con la simulación telemática se realiza mediante el estudio de las respuestas a 46 ítems de un cuestionario de aptitudes tipo Likert (ver Anexo 1). Los ítems se agrupan en seis bloques, denominados variables. Se considera como puntuación de un bloque o variable el total de las puntuaciones de los diferentes ítems o subvariables que componen el bloque. Las variables objeto de estudio son:

1) Variable utilidad

2) Variable nivel pre-tratamiento

3) Variable progreso en destrezas

4) Variable factores que influyen en el progreso

5) Variable contribución de las partes de la simulación telemática

6) Variable progreso en capacidades y actitud

Se han estimado las correlaciones muestrales de estas variables y se ha utilizado la metodología bootstrap (Gil, 2005) y las técnicas de inferencia clásica para determinar los intervalos de confianza (IC) de estas estimaciones y realizar un contraste de hipótesis acerca de la nulidad de estos coeficientes. El nivel de confianza de significación considerado es del 5\%. El paquete estadístico utilizado fue el sPSS versión 20.

\section{Análisis de los resultados}

Los resultados de las correlaciones simples entre las seis variables estudiadas se recogen en la tabla I. A modo explicativo, la matriz de correlaciones simples muestra las correlaciones momento producto de Pearson entre los distintos pares de variables. El rango de los coeficientes de correlación va de -1 a +1 y miden la fuerza de la relación lineal entre las variables. Un valor 0 indica relación lineal inexistente; un valor +1 relación lineal perfecta y directa, y un valor -1 relación lineal perfecta e inversa. El segundo número en cada bloque de la tabla I es un $p$-valor que indica el grado de significación de las correlaciones estimadas. Un p-valor por debajo de 0.05 indica que es posible rechazar la nulidad del coeficiente de correlación en la población con una probabilidad de error inferior al 5\%. En los resultados que se presentan se han obtenido intervalos de confianza para las correlaciones poblacional al 95\%, mediante la técnica bootstrap, obteniendo 10,000 remuestras a partir de la muestra original.

Como puede observarse, no se ha detectado en el estudio ninguna correlación significativa entre la variable utilidad y el resto de variables del cuestionario de tipo Likert analizadas. Los bajos valores de las correlaciones muestrales obtenidas reflejan que el simple hecho de reconocer la utilidad del inglés en la vida profesional no garantiza necesariamente que los sujetos acojan mejor la simulación telemática o que consideren que han alcanzado progresos más significativos en el dominio de las destrezas de la lengua inglesa. 


\begin{tabular}{|c|c|c|c|c|c|c|c|c|c|}
\hline \\
\hline & & & & Utilidad & $\begin{array}{l}\text { Nivel pre- } \\
\text { tratamiento }\end{array}$ & $\begin{array}{l}\text { Progreso } \\
\text { destrezas }\end{array}$ & $\begin{array}{l}\text { Factores } \\
\text { progreso }\end{array}$ & $\begin{array}{l}\text { Contribución } \\
\text { partes }\end{array}$ & $\begin{array}{l}\text { Proceso } \\
\text { capacidad- } \\
\text { actitud }\end{array}$ \\
\hline \multirow{7}{*}{ Utilidad } & \multicolumn{3}{|c|}{ Correlación de Pearson } & \multirow[t]{2}{*}{1} & \multirow{2}{*}{$\begin{array}{l}.086 \\
.552\end{array}$} & \multirow{2}{*}{$\begin{array}{l}.123 \\
.395\end{array}$} & \multirow{2}{*}{$\begin{array}{l}.262 \\
.066\end{array}$} & \multirow{2}{*}{$\begin{array}{l}.094 \\
.514\end{array}$} & \multirow{2}{*}{$\begin{array}{l}.138 \\
.339\end{array}$} \\
\hline & \multicolumn{2}{|c|}{ Sig. (bilateral) } & & & & & & & \\
\hline & $\mathrm{N}$ & & & \multirow{2}{*}{$\begin{array}{r}50 \\
0\end{array}$} & 50 & 50 & 50 & 50 & 50 \\
\hline & Bootstrap ${ }^{c}$ & Sesgo & & & & \multirow{2}{*}{$\begin{array}{r}-.002 \\
.133\end{array}$} & \multirow{2}{*}{$\begin{array}{r}-.004 \\
.124\end{array}$} & \multirow{2}{*}{$\begin{array}{l}.004 \\
.131\end{array}$} & \multirow{2}{*}{$\begin{array}{r}-.004 \\
.144\end{array}$} \\
\hline & & Típ. Error & & 0 & $\begin{array}{r}-.001 \\
.107\end{array}$ & & & & \\
\hline & & Intervalo de & Inferior & 1 & -.130 & -.153 & -.002 & -.163 & -.157 \\
\hline & & confianza al 95\% & Superior & 1 & .290 & .373 & .482 & .352 & .402 \\
\hline & Correlación de & earson & & .086 & 1 & -.252 & -.033 & $-.349^{*}$ & -.076 \\
\hline & Sig. (bilateral) & & & .552 & & .077 & .013 & .013 & .599 \\
\hline & $\mathrm{N}$ & & & 50 & 50 & 50 & 50 & 50 & 50 \\
\hline $\begin{array}{l}\text { Nivel } \\
\text { ncetcatamiento }\end{array}$ & Bootstrap ${ }^{c}$ & Sesgo & & -.001 & 0 & -.003 & .001 & .001 & -.005 \\
\hline & & Típ. Eгror & & .107 & 0 & .124 & .144 & .144 & .152 \\
\hline & & Intervalo de & Inferior & -.130 & 1 & -.487 & -.613 & -.613 & -.366 \\
\hline & & confianza al 95\% & Superior & .290 & 1 & -.006 & -.053 & -.053 & .225 \\
\hline & Correlación de & earson & & .123 & -.252 & 1 & $.448^{* *}$ & $.747^{\text {** }}$ & $.616^{* \pi}$ \\
\hline & Sig. (bilateral) & & & .395 & .077 & & .001 & .000 & .000 \\
\hline & $\mathrm{N}$ & & $x$ & 50 & 50 & 50 & 50 & 50 & 50 \\
\hline $\begin{array}{l}\text { Progreso } \\
\text { destrezas }\end{array}$ & Bootstrap $^{c}$ & Sesgo & & -.002 & -.003 & 0 & -.005 & -.001 & -.002 \\
\hline & & Típ. Error & & .133 & .124 & 0 & .119 & .051 & .079 \\
\hline & & Intervalo de & Inferior & -.153 & -.487 & 1 & .189 & .633 & .448 \\
\hline & & confianza al 95\% & Superior & .373 & -.006 & 1 & .656 & .835 & .754 \\
\hline & Correlación de & earson & $a$ & .262 & -.033 & $.448^{x-x}$ & 1 & $.425^{\text {*x }}$ & $.358^{*}$ \\
\hline & Sig. (bilateral) & & & .066 & .821 & .001 & & .002 & .011 \\
\hline & $\mathrm{N}$ & & & 50 & 50 & 50 & 50 & 50 & 50 \\
\hline Factores & Bootstrapc & Sesgo & & -.004 & -.004 & -.005 & 0 & -.002 & -.002 \\
\hline & & Típ. Еггог & & .124 & .147 & .119 & 0 & .123 & .110 \\
\hline & & Intervalo de & Inferior & -.002 & -.334 & .189 & 1 & .163 & .132 \\
\hline & & confianza al 95\% & Superior & .482 & .238 & .656 & 1 & .643 & .562 \\
\hline & Correlación de & earson & & .094 & $-.349^{x}$ & $.747^{\text {*i }}$ & $.425^{\text {k* }}$ & 1 & $.586^{* \pi}$ \\
\hline & Sig. (bilateral) & & & .514 & .013 & .000 & .002 & & .000 \\
\hline & $\mathrm{N}$ & & & 50 & 50 & 50 & 50 & 50 & 50 \\
\hline Contribución & Bootstrap $^{c}$ & Sesgo & & .004 & .001 & -.001 & -.002 & 0 & -.003 \\
\hline & & Típ. Еггог & & .131 & .144 & .051 & .123 & 0 & .085 \\
\hline & & Intervalo de & Inferior & -.1633 & -.613 & .633 & .163 & 1 & .398 \\
\hline & & confianza al 95\% & Superior & .352 & -.053 & .835 & .643 & 1 & .732 \\
\hline & Correlación de & earson & & .138 & -.076 & $.616^{\text {** }}$ & $.358^{*}$ & $.586^{* x}$ & 1 \\
\hline & Sig. (bilateral) & & & .339 & .599 & .000 & .011 & .000 & \\
\hline Progreso & $\mathrm{N}$ & & & 50 & 50 & 50 & 50 & 50 & 50 \\
\hline capacidad- & Bootstrap $^{c}$ & Sesgo & & -.004 & -.005 & -.002 & -.002 & -.003 & 0 \\
\hline actitud & & Típ. Error & & .144 & .152 & .079 & .110 & .085 & 0 \\
\hline & & Intervalo de & Inferior & -.157 & -.366 & .448 & .132 & .398 & 1 \\
\hline & & confianza al 95\% & Superior & .402 & .225 & .754 & .562 & .732 & 1 \\
\hline
\end{tabular}

“ 'La correlación es significante al nivel (bilateral). " " La correlación es significativa al nivel 0.01 (bilateral). ' Los resultados se basan en 10,000 muestras bootstrap. 
El coeficiente de correlación entre la variable nivel pre-tratamiento y la variable progreso en destrezas roza el nivel de significación establecido del $5 \%(r=-0.2524 p=0.0770)$ aunque la relación recogida es inversa. El intervalo de confianza bootstrap al $95 \%$ no contiene el valor 0 . Es decir, la conciencia de mejora en las destrezas es más elevada entre los sujetos cuyo nivel de inglés es más bajo en el pre-tratamiento, lo cual se observa en la figura 1.

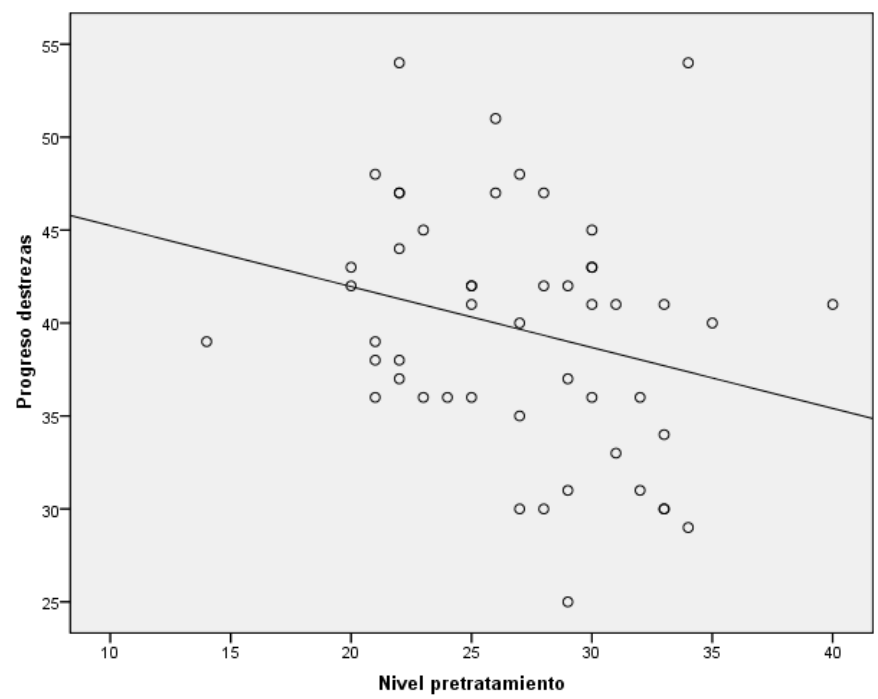

Figura 1. Dispersión de las variables progreso en destrezas y nivel pre-tratamiento

No existe correlación significativa entre la variable nivel pre-tratamiento y la variable factores que influyen en el progreso ( $r=-0.0329 \mathrm{p}=0.8205)$. Así como tampoco existe correlación significativa entre la variable nivel pre-tratamiento y la variable progreso en capacidades y actitud ( $r=-0.0762$ $p=0.5990$ ). Esto indica que no existe relación entre el nivel pre-tratamiento y los factores que considera el sujeto que son fundamentales en su progreso (motivación, número reducido de participantes, trabajo en equipo, clases participativas, etc.). Tampoco existe relación entre la variable nivel pre-tratamiento y la variable mejora en capacidades y actitud (capacidad de debate, negociación, toma de decisiones, o mejora en el interés y actitud). Posiblemente los factores que influyen en el progreso y la mejora en capacidades y cambio de actitud vayan más unidos a la adaptabilidad del sujeto a metodologías que se centran en la interacción social, que a su nivel de inglés pre-tratamiento.

En la figura 2 se observa un coeficiente de correlación significativo negativo ( $r=-0.3488 p=0.0131$ ) entre las variables nivel pre-tratamiento y contribución de las partes de la simulación telemática. 


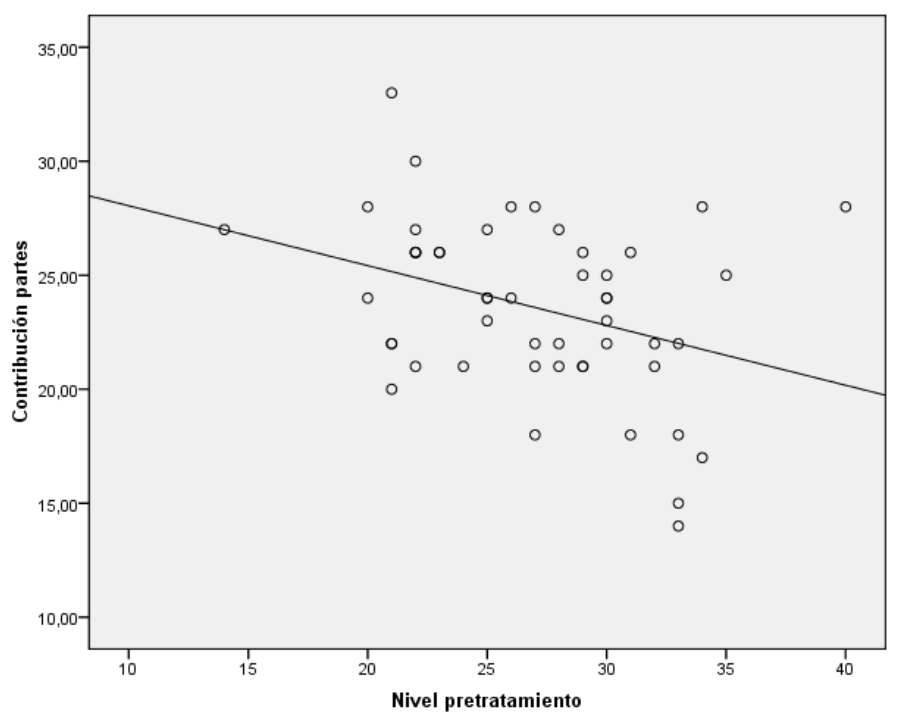

Figura 2. Dispersión de las variables contribución de las partes de la simulación telemática y nivel pre-tratamiento

La figura muestra que hay sujetos con alto nivel de inglés pre-tratamiento que consideran que algunas de las partes de la simulación contribuyen poco a su mejora. Del mismo modo, hay sujetos con un nivel pre-tratamiento relativamente más bajo que consideran que la contribución de las diferentes partes de la simulación telemática es muy notable.

Por otro lado, en la figura 3 se recogen también coeficientes de correlación significativos entre la variable progreso en destrezas y la variable factores que influyen en el progreso $\left(r=0.448^{* *}\right.$ $p=0.0011)$.

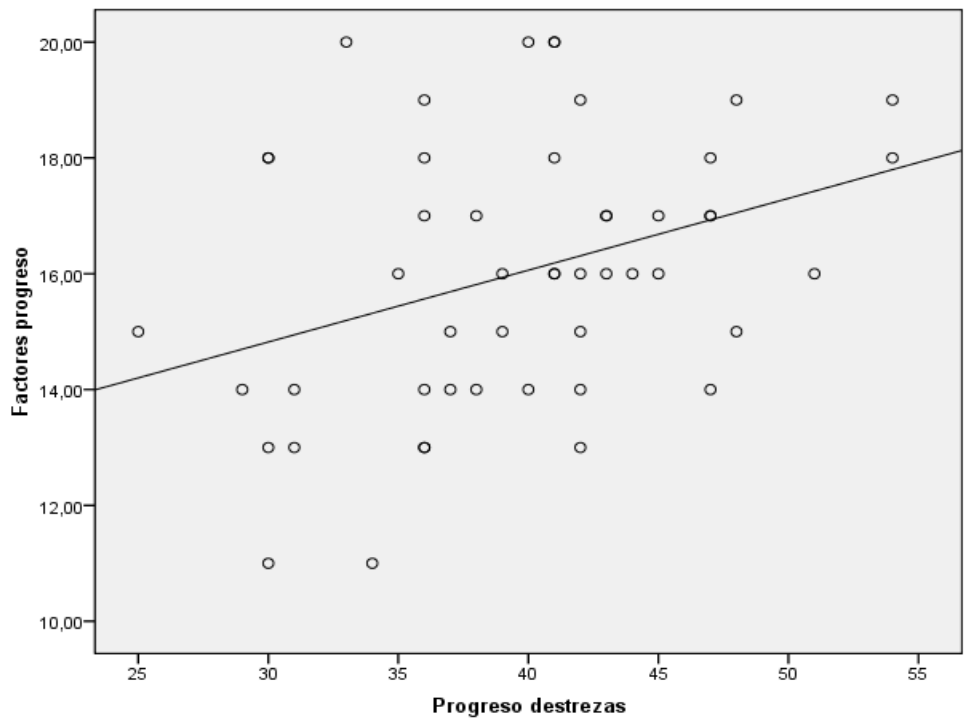

Figura 3. Dispersión de las variables factores que influyen en el progreso y progreso en destrezas

Los resultados muestran que los sujetos que perciben mejoras en la comprensión oral y escrita, en la expresión oral y escrita, en el nivel de fluidez y confianza durante la simulación telemática y 
en el manejo de un amplio registro de vocabulario son aquellos que valoran positivamente los factores que contribuyen a estos avances: la propia motivación, el número de sujetos por clase, la comunicación y participación activa en clase, el trabajo en equipo, la relación cercana con el profesor, la utilización de tecnologías de la información y comunicación.

Se observa una elevada correlación significativa positiva entre las variables progreso en destrezas y contribución de las partes de la simulación telemática $\left(r=0.7467^{* \star} \quad \mathrm{p}<0.0001\right)$, registrada en la figura 4.

Los resultados evidencian que los sujetos que experimentan mejoras en las destrezas de la lengua inglesa: hablar, escribir, leer, escuchar e interactuar, son los que consideran las distintas partes de la simulación telemática como los factores que contribuyen a tales avances.

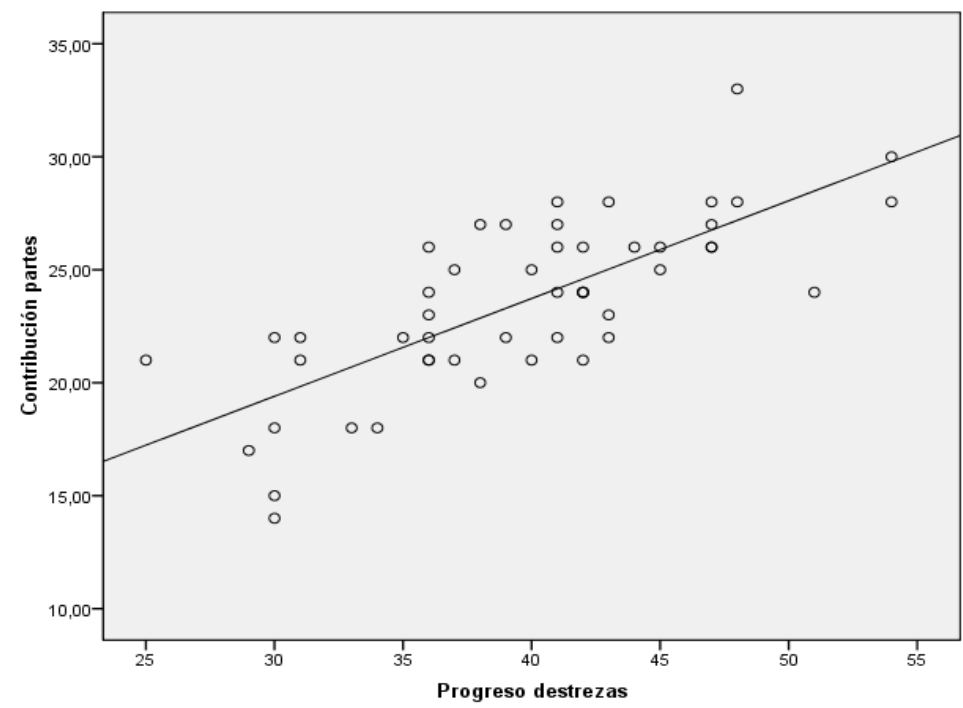

Figura 4. Dispersión de las variables contribución de las partes de la simulación telemática y progreso en destrezas

Se aprecia también una correlación muy significativa entre la variable progreso en destrezas y la variable progreso en capacidades y actitud $\left(r=0.6163^{* *} p<0.001\right)$ que se recoge en la figura 5. 


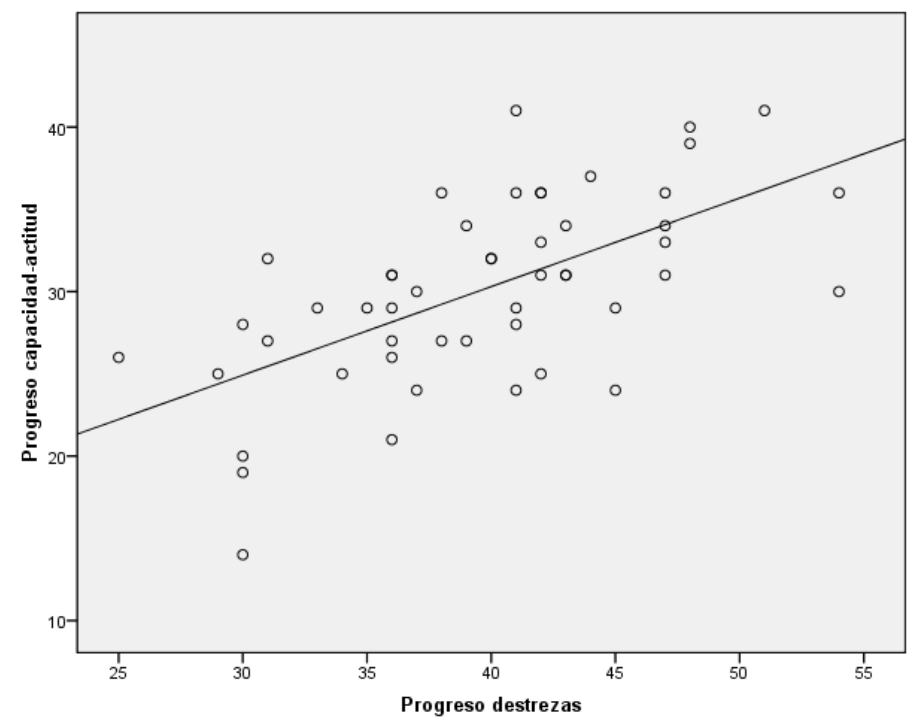

Figura 5. Dispersión de las variables progreso en capacidades y actitud y progreso en destrezas

La figura indica que los sujetos que mejoran sus conocimientos de la lengua inglesa en términos de habilidades lingüísticas mejoran también su capacidad para debatir, negociar, relacionarse y perciben un cambio de actitud respecto a la clase de inglés tradicional.

La figura 6 muestra que existe correlación significativa positiva entre la variable factores que influyen en el progreso y la variable contribución de las partes de simulación telemática $\left(r=0.4247^{* *}\right.$ $\mathrm{p}=0.0021$ ).

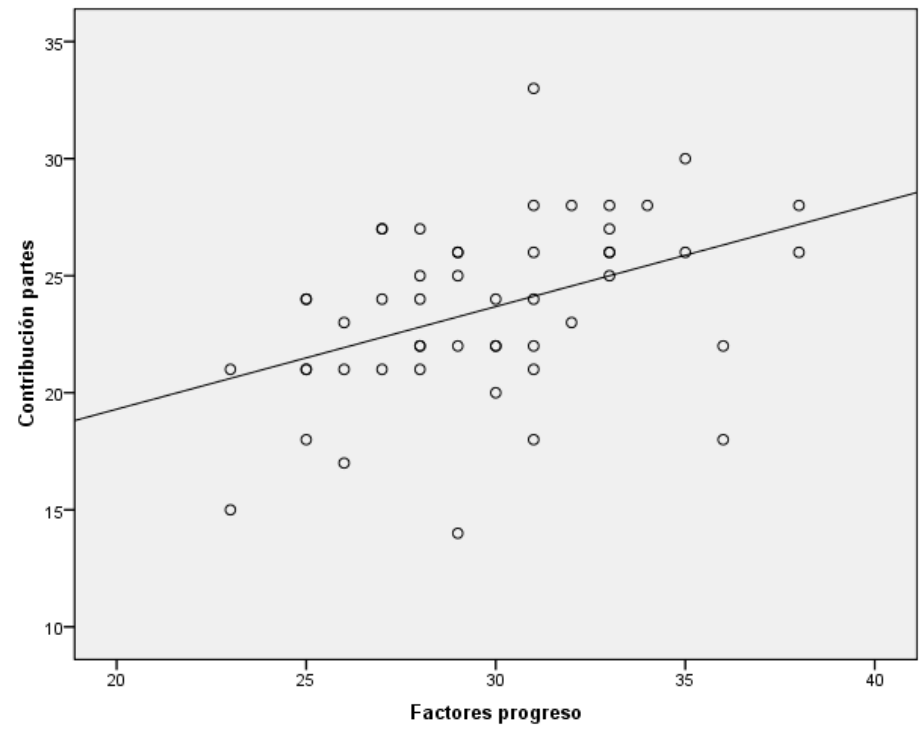

Figura 6. Dispersión de las variables contribución de las partes de la simulación telemática y factores que influyen en el progreso

De igual forma, existen coeficientes de correlación significativos entre la variable factores que influyen en el progreso y la variable progreso en capacidades y actitud ( $r=0.3576^{*} \quad \mathrm{p}=00108$ ) (ver figura 7$)$. 


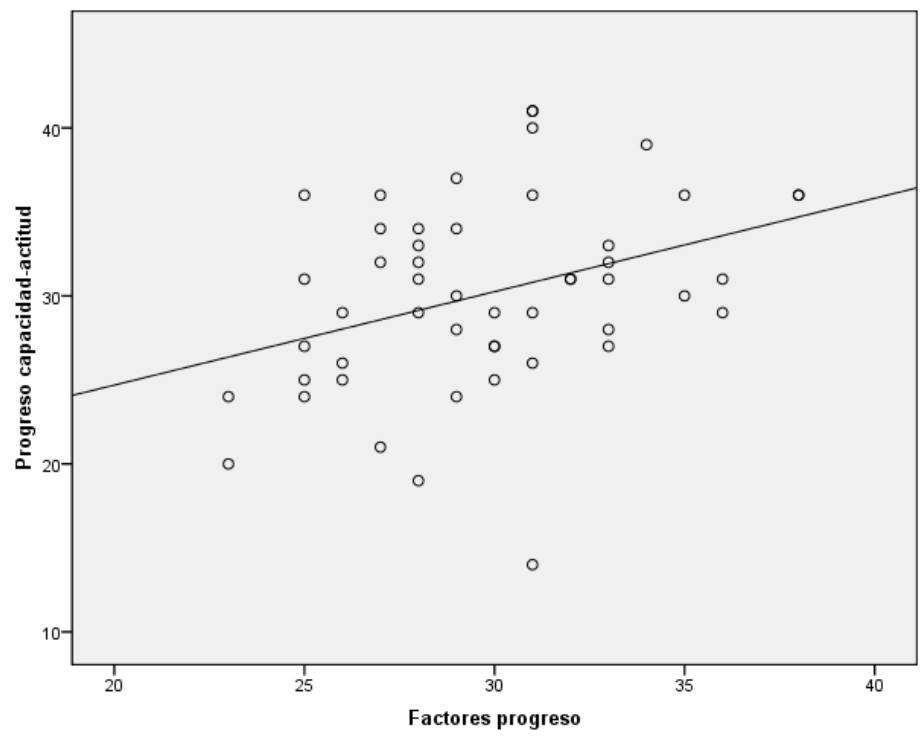

Figura 7. Dispersión de la variable progreso en capacidades y actitud y contribución de las partes de la simulación telemática

Por último, se observa que existe una correlación significativa positiva entre la variable contribución de las partes de simulación telemática y la variable progreso en capacidades y actitud $\left(\left\ulcorner=0.5857^{* *} \mathrm{p}<0.0001\right)\right.$ (ver figura 8).

Se puede afirmar, en este caso, que los sujetos que valoran significativamente el aporte que las partes de la simulación telemática hacen al aprendizaje del inglés, son aquellos que perciben mejoras en su capacidad para debatir, negociar, tomar decisiones y relacionarse con los demás. Además, estos sujetos son los que han cambiado su actitud respecto a la asignatura de inglés.

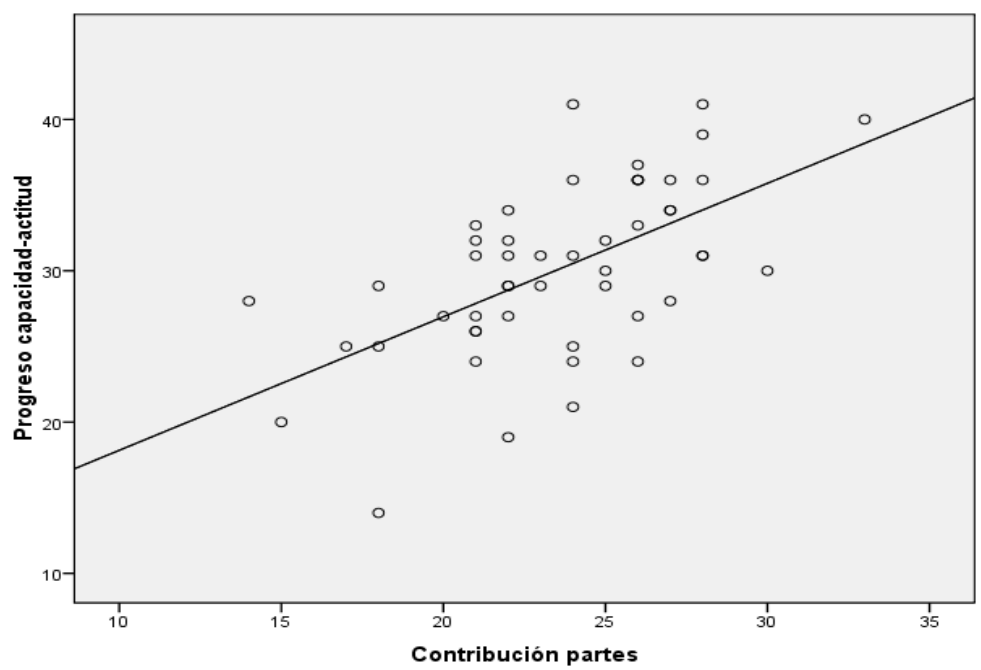

Figura. 8. Dispersión de la variable progreso en capacidades y actitud y contribución de las partes de la simulación telemática 


\section{Conclusiones}

El estudio de correlaciones entre variables indica que existe una fuerte correlación entre la simulación telemática y el progreso en destrezas lingüísticas. Existe también correlación significativa entre la simulación telemática y el progreso en capacidades transversales, y entre el progreso en destrezas y el progreso en capacidades transversales. Asimismo, se observa una correlación significativa inversa entre el nivel pre-tratamiento y la simulación telemática, siendo este método más valorado por los sujetos que tienen un nivel inferior en inglés. Es decir, la conciencia de mejora la manifiestan aquellos sujetos cuyo nivel de inglés es más bajo principalmente en la expresión oral. Se ha podido comprobar también que aquellos factores que inciden mayoritariamente en el progreso y la mejora en capacidades y cambio de actitud, son aquellos relacionados con la adaptabilidad del sujeto a metodologías de interacción social. Se ha detectado que sujetos con alto nivel de inglés pre-tratamiento consideran que algunas de las partes de la simulación contribuyen poco a su mejora. Sin embargo, para la mayoría del estudiantado lo que realmente consigue el progreso en destrezas lingüísticas son las partes de la simulación, incluso más que los factores colaterales como el trabajo cooperativo, la relación cercana profesor-alumno, las clases participativas o la utilización de tecnologías de la información y comunicación. Los aspectos lingüísticos donde los sujetos han percibido mayor progreso son la comprensión oral y escrita, la expresión oral y escrita, el nivel de fluidez y confianza o el manejo de vocabulario específico para cada temática.

Se puede deducir que los sujetos que mejoran sus conocimientos de la lengua inglesa en términos de habilidades lingüísticas $y$, teniendo en cuenta las exigencias de la simulación telemática, mejoran también su capacidad para debatir, negociar, relacionarse y perciben un cambio de actitud respecto a la clase de inglés tradicional.

Por último, cabe resaltar que la concienciación de la futura utilidad del inglés en la vida profesional no es suficiente para garantizar la aceptación de la metodología y el progreso en destrezas. Futuros estudios se centrarán en determinar la correlación existente entre las subvariables mediante un análisis de correlaciones; dimensionalidad del cuestionario mediante un análisis de componentes principales; la consistencia interna del cuestionario mediante el coeficiente alpha de Cronbach; la identificación de variables y sub-variables asociadas a la satisfacción con la experiencia mediante un análisis de regresión.

\section{Referencias}

Angelini, M. L. (2012). La simulación y juego en el desarrollo de las destrezas de producción en lengua inglesa (Tesis doctoral). Universitat Politècnica de València. doi:10.4995/Thesis/10251/16011

Angelini, M. L. y García-Carbonell, A (2014). Análisis cualitativo sobre la simulación telemática como estrategia para el aprendizaje de lenguas. Revista Iberoamericana de Educación, 64(2), 1-15.

Angelini, M. L., García-Carbonell, A. y Watts, F. (2014). Student perceptions of gain in telematic simulation. En W. C. Kriz (Ed.), The shift from teaching to learning: individual, collective and organizational learning through gaming simulation (pp. 176-186). Austria: WB Verlag.

Asal, V. y Blake, E. L.(2006). Creating simulations for political science education. Journal of Political Science Education 2(1), 1-18. doi:10.1080/15512160500484119 
Blum, A. y Scherer, A. (2007, febrero). What creates engagement? An analysis of student participation in ICONS simulations. En APSA Teaching and Learning Conference. Carolina del Norte: American Political Science Association.

Bredemeier, M. E. y Greenblat, C. (1981). The educational effectiveness of simulation games. Simulation \& Games 12(3), 307-332. doi:10.1177/104687818101200304

Cherryholmes, C. H. (1966). Some current research on effectiveness of educational simulations: implications for alternative strategies. American Behavioral Scientist, 10(2), 4-7.

doi:10.1177/000276426601000202

Crookall, D. (2010). Serious games, debriefing, and simulation/gaming as a discipline. Simulation \& Games, 41(6), 898-920. doi:10.1177/1046878110390784

Crookall, D. y Arai, K. (Eds.) (1995). Simulation and gaming across disciplines and cultures. California: Sage.

Crookall, D. y Oxford R. L. (Eds.) (1990). Simulation, gaming, and language learning. Nueva York: Newbury House Publishers.

De Caluwé, L., Hofstede, G. J. y Peters, V. (Eds.) (2008). Why do games work? Amsterdam: Kluwer.

Degarmo, D. (2006, febrero). ICONS and resistant populations: assessing the impact of the International Communication and Negotiation Simulations Project on Student Learning at SIUE. En APSA Teaching and Learning Conference. Washington: American Political Science Association.

Druckman, D. y Ebner, N. (2008). Onstage or behind the scenes? Relative learning benefits of simulation role-play and design. Simulation \& Gaming 39(4): 465-497.

doi:10.1177/1046878107311377

Duke, D. (1974). Gaming: the future’s language. Nueva York: Sage.

Duke, D. y Geurts, J. (2004). Policy games for strategic management: pathways into the unknown. Amsterdam: Dutch University Press.

Ekker, K. (2000). Changes in attitude towards simulation based distributed learning. En B. Wasson, F. Guribye y A. Mørch (Eds.), Project DoctA: Design and use of Collaborative Tele-learning Artefacts (pp. 112-120). Noruega: Unipub forlag. Recuperado de http://www.ituarkiv.no/filearchive/fil ITU Rapport 05.pdf

Ekker, K. (2004). User satisfaction and attitudes towards an internet-based simulation. En Kinshuk, D. G. Sampson y P. Isaías (Eds.), Proceedings of the IADIS International Conference on Cognition and Exploratory Learning in Digital Age (pp.224-232). Recuperado de http://citeseerx.ist.psu.edu/viewdoc/download?doi=10.1.1.108.9485\&rep=rep1\&type=pdf

Ekker, K. y Eidsmo, A. (2006, mayo). Evaluation of Software used in an Internet-based Simulation: Are there any Cultural differences? En Congreso IADIS e-Learning. Recuperado de http://citeseerx.ist.psu.edu/viewdoc/download;jsessionid=FE274986456C6DE037B9EC1B36E893 84?doi=10.1.1.138.1971\&rep=rep1\&type=pdf

Ekker, K. y Sutherland, J. (2005). Telematic simulations and changes in attitudes towards simulation topics. En G. Richards (Ed.), Proceedings of World Conference on E-Learning in Corporate, Government, Healthcare, and Higher Education (pp. 2034-2041). EU: AACE. 
Ekker, K. y Sutherland, J. (2009). Simulation game as a learning experience: An analysis of learning style. En Kinshuk, D. G. Sampson, J. M. Spector, P. Isaías y D. Ifenthaler (Eds.), Proceedings of the IADIS International Conference on Cognition and Exploratory Learning in Digital Age (pp. 291-312). Italia: IADIS.

García-Carbonell, A. (1998). Efectividad de la simulación telemática en el aprendizaje del inglés técnico (Tesis doctoral). Universitat de Vàlencia.

García-Carbonell, A., Andreu-Andrés, A. y Watts, F. (2014). Simulation and gaming as the future's language of language learning and acquisition of professional competences. En R. D. Duke y W. C. Kriz (Eds.), Back to the future of gaming (pp. 214-228). Austria: WB Verlag.

García-Carbonell, A. y Watts, F. (2012). Investigación empírica del aprendizaje con simulación telemática. Revista Iberoamericana de Educación 59(30), 1-11. Recuperado de http://rieoei.org/rie contenedor.php?numero=4395\&titulo=Investigaci\%C3\%B3n\%20emp\%C3\% ADrica\%20del\%20aprendizaje\%20con\%20simulaci\%C3\%B3n\%20telem\%C3\%A1tica

García-Carbonell, A.; Watts, F. y Andreu-Andrés, M. A. (2012). Simulación telemática como experiencia de aprendizaje de la lengua inglesa. Revista de Docencia Universitaria. 10(3), 301-323. Recuperado de http://red-u.net/redu/index.php/REDU/issue/view/69

Garrido-Iñigo, P. (2012). Viabilidad de las plataformas virtuales en la enseñanza de una lengua extranjera. Revista de Investigación en Educación, 10(2), 129-140. Recuperado de http://reined.webs.uvigo.es/ojs/index.php/reined/article/view/499

Gil, J. (2005). Aplicación del método bootstrap al contraste de hipótesis en la investigación educativa. Revista de Educación 336, 251-265. Recuperado de http://www.mecd.gob.es/revistade-educacion/numeros-revista-educacion/numeros-anteriores/2005/re336/re336 14.html

Goodman, F. L. (1984). The computer as plaything. Simulation \& Gaming 15(1), 65-73.

doi:10.1177/0037550084151004

Goodman, F. L. (1995). Practice in theory. Simulation \& Gaming, 26(2), 178-190.

doi:10.1177/1046878195262005

Gredler, M. (1992). Designing and evaluating games and simulations. A Process Approach. Londres: Kogan Page.

Greenblat, C. S.(1975). Teaching with simulations games: a review of claims and evidence. En C. Greenblat y R. D. Duke (Eds.), Gaming-simulation: rationale, design and applications (pp. 270-284). Nueva York: Sage.

Greenblat, C. S., Duke, R. D. (1975). Gaming-simulation: rationale, design and applications. Nueva York: Sage.

Greenblat, C. S. y Duke, D. (1981). Principles and practices of gaming-simulation. California: Sage.

Halleck, G. y Coll-García, J. (2011). Developing problem-solving and intercultural communication: An online simulation for engineering students. Journal of Simulation/Gaming for Learning and Development 1(1), 1-12. Recuperado de http://www.thaisim.org/sgld/sgld online-first/sgld halleck intercultural-engineering olf.pdf

Hofstede, G. J., Caluwé, L. y Peters, V. (2010). Why simulation games work-in search of the active substance: a synthesis. Simulation \& Gaming, 41(6), 824-843. doi:10.1177/1046878110375596 
Klabbers, J. (2009). The magic circle: principles of gaming simulation. Países Bajos: Sense.

Kolb, D. A. (1984). Experiential learning: experience as the source of learning. NJ: Prentice-Hall.

Kolb, A. Y. y Kolb, D. A. (2005a). Learning styles and learning spaces: Enhancing experiential learning in higher education. Academy of Management Learning and Education 4(2), 193-212. doi:10.5465/AMLE.2005.17268566

Kolb, A. Y. y Kolb, D. A. (2005b). The Kolb learning style inventory-version 3.12005 Technical specifications. Massachusetts: Hay Group.

Kolb, A. Y. y Kolb; D. A. (2009). The learning way: meta-cognitive aspects of experiential learning. Simulation \& Gaming 40(3), 297-327. doi:10.1177/1046878108325713

Kriz, W. C. y Hense, J. U. (2006). Theory-oriented evaluation for the design of and research in gaming and simulation. Simulation \& Gaming, 37(2), 268-283. doi:10.1177/1046878106287950

Lay, C. y Smarick, K. (2006). Simulating a senate office: the impact on student knowledge and attitudes. Journal of Political Science Education 2(2), 131-146. doi:10.1080/15512160600668967

Lederman, L. C. (1983). Differential learning outcomes in an instructional simulation: Exploring the relationship between designated role and perceived learning outcome. Communication Quarterly 31(4), 266-270. doi: 10.1080/01463378309369514

Lederman, L. C. (1992). Debriefing: towards a systematic assessment of theory and practice. Simulation \& Gaming, 23(2), 145-160. doi:10.1177/1046878192232003

Lederman, L. C. y Steward, L. (1991). The rules of the game. Simulation \& Gaming, 22(4), 502-507. doi:10.1177/1046878191224007

MacDonald, P.; García-Carbonell; A. y Carot, J. M. (2013). Computer learner corpora: analysing interlanguage errors in synchronous and asynchronous communication. Language Learning \& Technology, 17(2), 36-56. Recuperado de http://lt.msu.edu/issues/june2013/macdonaldetal.html

Morales, P. (2010). Investigación e innovación educativa. Revista Iberoamericana sobre Calidad, Eficacia y Cambio en Educación 8(20, 47-73. Recuperado de

http://www.rinace.net/reice/numeros/arts/vol8num2/art3.htm

Morales, P. (2012). El Profesor en la era de las competencias. Madrid: Universidad Pontificia de Comillas.

Rising, B. (1999). La eficacia didáctica de los juegos de simulación por ordenador en el aprendizaje del Inglés como lengua extranjera en alumnos de Derecho, Económicas e Ingeniería (Tesis doctoral). Madrid: Universidad Pontificia de Comillas.

Rising, B. (2009). Business simulations as a vehicle for language acquisition. En V. Guillén-Nieto, C. Marimón-Llorca y C. Vargas-Sierra (Eds.), Intercultural Business Communication and Simulation and Gaming Methodology (pp. 317-339). Suiza: Peter Lang,

Shubik, M. (1994). Some musings on gaming and simulation. Simulation \& Games, 25(2), 251-258. doi:10.1177/1046878194252015 
Shuman, L.; Besterfield-Sacre, M. y McCourty, L. (2005). The ABET "Professional skills"-can they be taught? Can they be assessed? Journal of Engineering Education 94(1), 41-55. doi: 10.1002/j.21689830.2005.tb00828.x

Taylor, J. L. y Walford, R. (1978). Learning and the simulation game. California: Sage.

Vernon, V. (2006, julio). An old-timer's reflections on IP simulations. En APSA Teaching and Learning Conference. Washington: American Political Science Association.

Watts, F., García-Carbonell, A. y Rising, B. (2011). Student perceptions of collaborative work in telematic simulation. Journal of Simulation/Gaming for Learning and Development, 1(1), 1-16. Recuperado de http://www.upv.es/diaal/publicaciones/sgld watts collaborative-telematics olf.pdf

Wedig, T. (2010). Getting the most from classroom simulations: strategies for maximizing learning outcomes. Ps: Political Science \& Politics, 43(3), 547-555.

doi:10.1017/S104909651000079X

Wolfe, J. y Crookall; D. (1998). Developing a scientific knowledge of simulation/gaming. Simulation \& Gaming, 29(1), 7-19. doi:10.1177/1046878198291002 


\section{Anexo 1}

\section{Cuestionario de aptitudes tipo Likert utilizado en el presente estudio}

\section{Variable 1- Utilidad}

1- ¿Crees que el inglés te será útil en tu futura vida profesional?

2-¿ंTienes interés personal por la lengua inglesa? (Visitar países de habla inglesa, hacer amigos, conocer su cultura, conocer la lengua para fines específicos)

\section{Variable 2- Nivel pre-tratamiento}

3- Nivel general de inglés.

4- Nivel de comprensión lectora (entender el discurso escrito).

5- Nivel de comprensión oral (entender el discurso hablado).

6- Nivel de expresión escrita (escribir, redactar).

7- Nivel de expresión oral (hablar).

8- ¿Tienes confianza en ti mismo/a para expresar tus ideas?

9- ¿Cuál era tu nivel de fluidez?

10- ¿Crees que eras capaz de comunicarte en inglés?

\section{Variable 3- Progreso oral}

11- ¿Crees que has mejorado la comprensión oral?

13- ¿Crees que has mejorado la expresión oral?

14- ¿Crees que tu capacidad para transmitir ideas ha mejorado?

15- ¿Crees que has adquirido fluidez mediante la simulación?

16- ¿Crees que has alcanzado mayor confianza en ti mismo/a para expresarte en inglés?

20- ¿Crees que has mejorado tu pronunciación?

21- ¿Crees que has mejorado tu entonación y ritmo propios de la lengua inglesa?

22- ¿Crees que ha mejorado tu capacidad de comunicación en la lengua inglesa?

\section{Variable 4- Progreso escrito y vocabulario}

12- ¿Crees que has mejorado la expresión escrita?

18- ¿Crees que has ampliado tu vocabulario?

19- ¿Crees que haces mayor uso del vocabulario con precisión?

\section{Variable 5- Factores que influyen en el progreso}

25- ¿Influye en el progreso que las clases sean participativas?

26- ¿Se fomenta el trabajo en equipo?

27- ¿Crees que la relación del profesor con los alumnos es más cercana?

28- ¿Crees que se fomenta la utilización de las tecnologías de la información y la comunicación?

\section{Variable 6-Contribución de las partes de la simulación telemática}

31- ¿Crees que la fase de información en la simulación ha mejorado tu nivel de inglés?

32- ¿Crees que la producción de mensajes y comunicados, propuestas y "reports" han contribuido a tu progreso en inglés?

33- ¿Crees que la lectura de correspondencia en inglés han contribuido a tu progreso en la lengua inglesa?

34- ¿Crees que los debates en tu grupo (intragrupo) han contribuido a tu progreso en la lengua inglesa?

35- ¿Crees que los debates entre todos los grupos (intergrupos) han contribuido a tu progreso en la lengua inglesa?

36- ¿Crees que las sesiones de puesta en común han contribuido a tu progreso en la lengua inglesa?

37- ¿Crees que la producción y presentación del documento final han contribuido a tu progreso en la lengua inglesa?

\section{Variable 7- Mejora en capacidades}

17- ¿Crees que has mejorado la organización del discurso oral de manera lógica y fácil de seguir? (Introducción, puntos principales, conclusión)

41- ¿Crees que la simulación ha mejorado tu capacidad de debate?

42- ¿Crees que la simulación ha mejorado tu capacidad de negociación?

43- ¿Crees que la simulación ha mejorado tu capacidad de toma de decisiones?

44- ¿Crees que la simulación ha mejorado tu capacidad de relacionarte con los demás?

Variable 8-Cambio de actitud hacia el aprendizaje del inglés

38- ¿Este año con la simulación, ha cambiado tu actitud respecto a la clase de inglés?

39- ¿La simulación ha aumentado tu interés en estudiar la asignatura?

40- ¿Crees que la simulación ha mejorado el clima del aula?

45- ¿La simulación te ha despertado otro tipo de intereses como: relacionarte con gente a través de intercambios académicos, participar en actividades académicas con soporte tecnológico (simulaciones telemáticas, conferencias, etc.)?

46- ¿Eres partidario de utilizar simulaciones en el aula? 